\title{
Properties of autonomous and injection locked flux flow oscillators
}

\author{
Mygind, Jesper; Koshelets, V. P.; Shchukin, A. V.; Shitov, S. V.; Lapytskaya, I. L.
}

Published in:

I E E E Transactions on Applied Superconductivity

Link to article, DOI:

10.1109/77.403211

Publication date:

1995

Document Version

Publisher's PDF, also known as Version of record

Link back to DTU Orbit

Citation (APA):

Mygind, J., Koshelets, V. P., Shchukin, A. V., Shitov, S. V., \& Lapytskaya, I. L. (1995). Properties of autonomous and injection locked flux flow oscillators. I E E E Transactions on Applied Superconductivity, 5(2), 2951-2954. https://doi.org/10.1109/77.403211

\section{General rights}

Copyright and moral rights for the publications made accessible in the public portal are retained by the authors and/or other copyright owners and it is a condition of accessing publications that users recognise and abide by the legal requirements associated with these rights.

- Users may download and print one copy of any publication from the public portal for the purpose of private study or research.

- You may not further distribute the material or use it for any profit-making activity or commercial gain

- You may freely distribute the URL identifying the publication in the public portal 


\title{
Properties of Autonomous and Injection Locked Flux Flow Oscillators
}

\author{
J. Mygind \\ Physics Department, Technical University of Denmark, B309, DK-2800 Lyngby, Denmark \\ V. P. Koshelets, A. V. Shchukin, S. V. Shitov, and I. L. Lapytskaya \\ Institute of Radio Engineering and Electronics, Russian Academy of Sciences, Moscow, Russia
}

\begin{abstract}
Flux flow oscillators (FFO) have been experimentally investigated at frequencies up to 850 GHz. At $440 \mathrm{GHz}$ the received power in an on-chip integrated SIS mixer is $5 \mu \mathrm{W}$. The first experimental measurements of the FFO linewidth as a function of applied dc bias current and magnetic field have been performed both for two autonomous FFOs and with one of the FFOs injection-locked to a narrow-band external microwave source. By beating the two autonomous FFOs an integral spectral linewidth as low as $750 \mathrm{kHz}$ is measured at $280 \mathrm{GHz}$. Mechanisms leading to a broadening of the FFO linewidth are discussed. Experimentally it is shown that the FFO can be used as harmonic generator.
\end{abstract}

\section{INTRODUCTION}

The uni-directional and viscous flow of magnetic flux quanta in a long (length, $L \gg \lambda_{J}$, the Josephson penetration depth) Josephson tunnel junction with high damping [1] has recently been successfully used in the development of local oscillators (LO) for fully superconducting integrated submillimeter wave SIS receivers [2]-[6]. The frequency of this so called Flux Flow Oscillator (FFO) can be tuned over a wide frequency range, limited only by the superconductor gap frequency. Moreover, the FFO provides sufficient output power to pump a SIS mixer array detector. Preliminary FFO spectral linewidth measurements have demonstrated encouraging values: $130 \mathrm{kHz}$ at $70 \mathrm{GHz}$ [2], about $1 \mathrm{MHz}$ at $140 \mathrm{GHz}$ [5] and $2.1 \mathrm{MHz}$ at $320 \mathrm{GHz}[6]$.

\section{EXPERIMENTAL SYSTEM}

For comprehensive studies of the FFO properties a series of different integrated circuits comprising long Josephson junctions and microwave SIS detectors have

Manuscript received October 18, 1994.

J. Mygind, e-mail myg@mips.fys.dtu.dk; fax (+45) 4593 1669; V. P. Koshelets, e-mail iree235@glas.apc.org; fax (+7) 0952038414

The work was supported in part by the Russian Program of Fundamental Research (G 92-02-3484), the Russian State Scientific Program "Superconductivity" (G 91009), NATO (LG 921040), and the Danish Research Academy. (a)

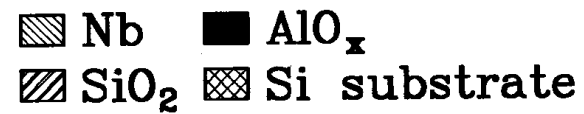

(b)
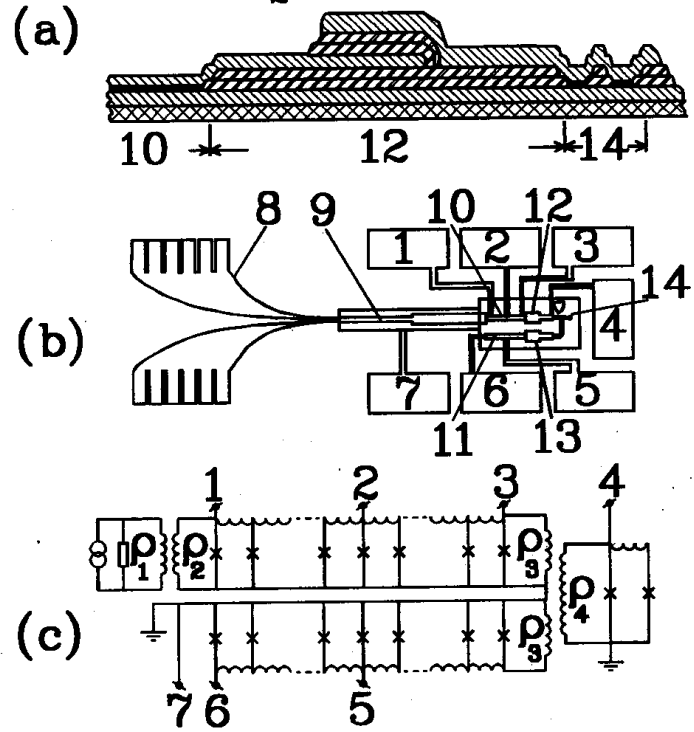

Fig. 1. Cross-section (a), schematic drawing (b) and simplified equivalent diagram (c) of the experimental on-chip integrated circuit. "1" - "7" - contact pads; "8" - fin-line antenna; "g", "12", "13" - Chebyshev impedance matching transformers; "10" - FFO1; "11" - FFO2; "14" - two-junction SIS array with tuning-out circuit.

been made. A cross-section (a), a layout (b) and a simplified equivalent diagram (c) of one of the tested on-chip integrated circuits are shown in Fig. 1.

The circuit comprises two identical FFOs (FFO1 - "10" and FFO2 - "11"; length, $L=200 \mu \mathrm{m}$, width, $W=$ $1.5 \mu \mathrm{m})$, a SIS mixer array detector with a capacitancetune-out circuit (" 14 ", junction area, $S=1.3 * 1.3 \mu \mathrm{m}^{2}$ ), three impedance matching transformers (" $9 ", " 12$ " and "13"), and a fin-line antenna (" 8 "). The tuning-out inductances for the two SIS junction (parallel dc biased [7]) array ("14") and the transformers ("12", "13") were designed for optimum performance at $350 \mathrm{GHz}$. All microcircuits had three superconducting $\mathrm{Nb}$ layers and two double insulating $\mathrm{SiO}$ layers. The integrated circuits have been fabricated with a technique developed for producing SIS mixer elements and Rapid Single Flux Quantum (RSFQ) digital devices [8]. Details of the circuit design

$1051-8223 / 95 \$ 04.00$ C 1995 IEEE 


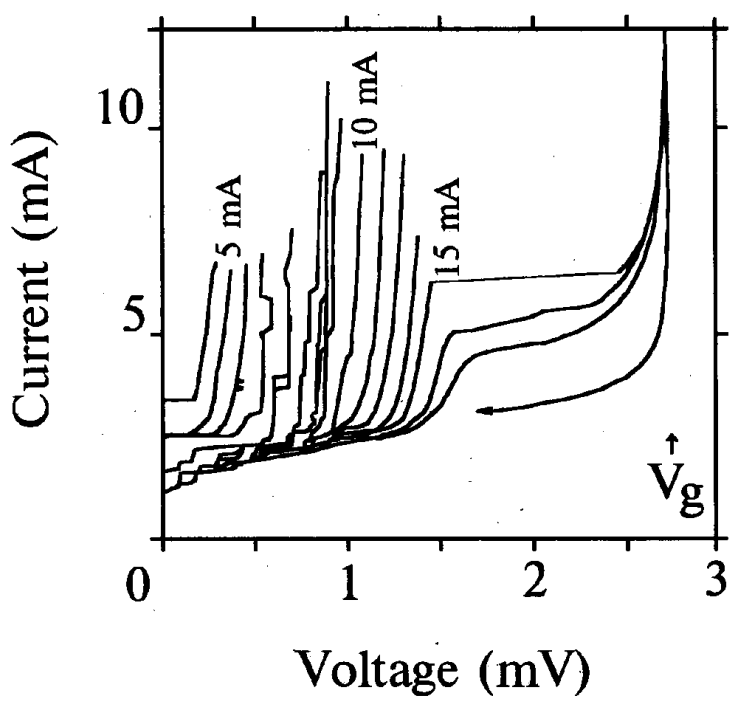

Fig. 2. $I V$-curves of the FFO at different magnetic fields produced by the control line current, circuit "HF".

and experimental set-up will be described elsewhere [9]. The high quality $\mathrm{Nb}-\mathrm{AlO}_{x}-\mathrm{Nb}$ tunnel junctions have a critical current density, $j_{c}=8 \mathrm{kA} / \mathrm{cm}^{2}$ and a Josephson penetration depth, $\lambda_{j}=4 \mu \mathrm{m}$. The product of the normal state resistance and the junction area, $R_{n} * S$, is $25 \Omega \mu \mathrm{m}^{2}$.

For the investigation of the properties of the autonomous FFO we used two different test circuits made on the same chip with identical FFOs (length, $L=500 \mu \mathrm{m}$, width, $W=4.5 \mu \mathrm{m}$ ) and SIS mixer array detectors (area, $S=1.1 * 1.1 \mu \mathrm{m}^{2}$ ). The tuning-out inductances for the two-junction SIS array (connected in parallel both for $\mathrm{dc}$ and rf currents) and the two-step impedance transformers were different for the two test circuits. One (named WB) had a fairly low center frequency of $400 \mathrm{GHz}$ and wide band frequency band $( \pm 250 \mathrm{GHz})$ while the other (named HF) had a higher center frequency but smaller bandwidth $(600 \mathrm{GHz} \pm 100 \mathrm{GHz})$.

\section{Properties of the autonomous FFOs}

A typical FFO $I V$-curve (IVC) recorded at different values of the magnetic field, produced by a dc current, $I_{m}$, in a control line, are shown in Fig. 2. The control line was integrated in the base electrode of the FFO. We used a low-value resistor $(\mathrm{R} \leq 1 \Omega)$ incorporated in the base electrode to "disconnect" the superconducting films and allow for the magnetic field to enter mainly perpendicular to the junction. The test circuits had very similar IVCs. The FFOs were designed with one end specially taylored (length about $50 \mu \mathrm{m}$ ) in order to steepen the flux flow steps [1], [6], and both FFOs showed the asymmetric $I_{c}\left(I_{m}\right)$ interference pattern typical for long overlap junctions with a uniform dc bias current distribution. The

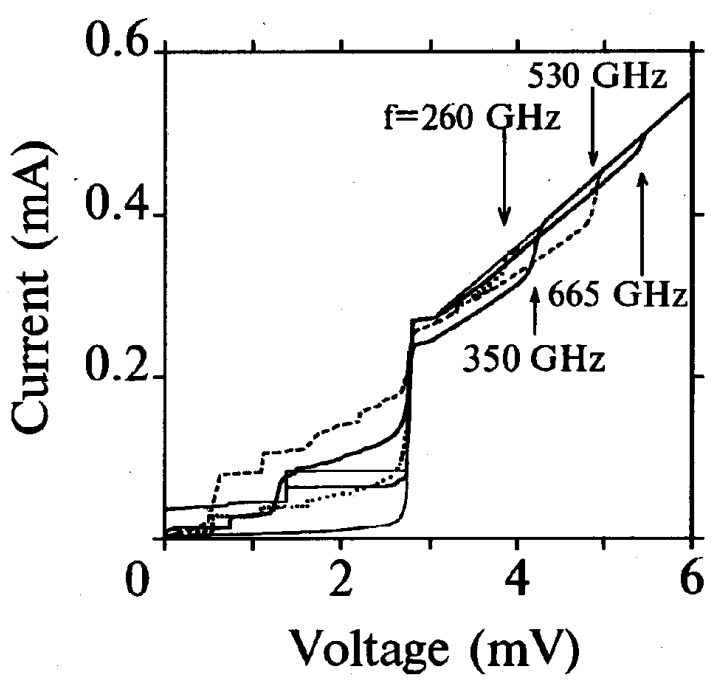

Fig. 3. IVCs of the SIS detector (circuit "WB") pumped by the FFO at different frequencies.

voltage separation between the tiny Fiske steps barely observable at very low voltages in Fig. 2 is $22 \mu \mathrm{V}$.

The peculiarities of the IVCs at voltages below $900 \mu \mathrm{V}$, which are seen in all our FFOs, are fully reproducible and might be due to internal resonant phenomena. They are also seen in FFOs not equipped with transformers and detectors. Due to self-inductance the integrated thin-film resistor, which shunts the full length of the FFO, provides a shunt loss damping parameter, $\alpha \approx 0.2$, only up to around $200 \mathrm{GHz}$. Above this frequency $\alpha \approx 0.01$ so even with a normalized length, $l=L / \lambda_{j} \approx 100$ we were not in the "pure" flux flow regime until $V>900 \mu \mathrm{V}$ where the surface loss, $\beta$, of the superconducting films starts to play a dominant role. The junction quality factor $Q_{j}$ is strongly frequency dependent: $Q_{j}=1 /\left\{\left(f_{j} / f_{p 0}\right)^{2} \beta+\alpha\right\}$, where $f_{j}=V_{d c} / \Phi_{0}$ is the Josephson frequency in the bias point and $f_{p 0}$ is the maximum plasma frequency. $\Phi_{0}=$ $h /(2 e)$ is the magnetic flux quantum.

Numerous experiments in which FFO power was detected by different SIS elements coupled via other transformers have demonstrated that the FFO itself can oscillate in a very wide frequency range. The IVCs of the SIS detector (circuit WB) pumped by the FFO at different frequencies are shown in Fig. 3. One can see that there is enough power to pump the SIS mixer two-junction parallel array in a wide frequency range from $260 \mathrm{GHz}$ up to $665 \mathrm{GHz}$. The highest detectable frequency was $850 \mathrm{GHz}$. The dependence of the current of the first Shapiro step on the FFO frequency is shown in Fig. 4 for circuit HF. The SIS detector in this case was shunted by a $10 \Omega$ resistor in order to ease the detection of the Shapiro steps. The FFO power was kept constant by adjusting the bias so that the $I V$-product remained the same. Notice the Riedel peak at $635 \mathrm{GHz}$ corresponding to a gap voltage of $2.65 \mu \mathrm{V}$. 


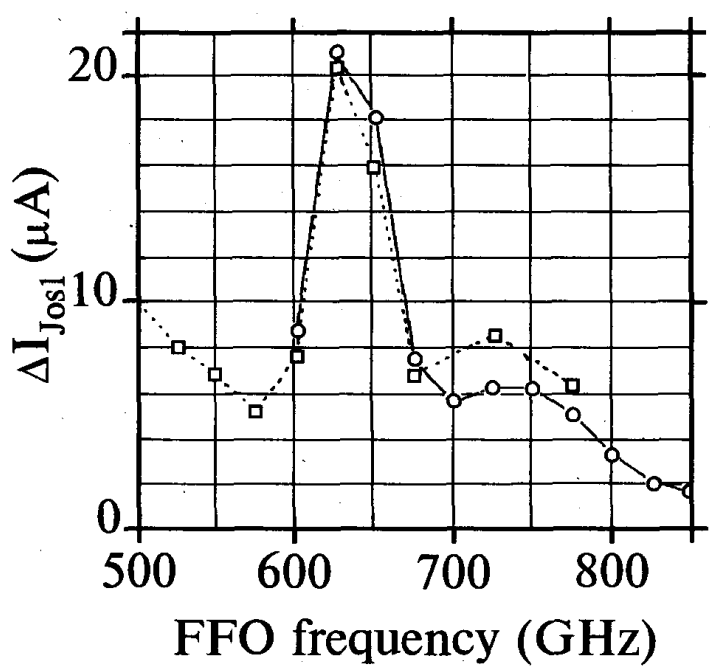

Fig. 4. Amplitude of the first Shapiro step of the SIS detector as a function of the FFO frequency (constant FFO power, test circuit HF).

The microwave power at a given frequency may be adjusted by simultaneously changing the dc bias current and the control current keeping the FFO voltage constant. We have measured the microwave power received by the SIS detector by recording its critical current and the variation of the Shapiro steps. At $440 \mathrm{GHz}$ it was possible to suppress the critical current $\left(I_{c}=1.3 \mathrm{~mA}, R_{n}=2.2 \Omega\right)$ to zero and measure the maximum amplitude of the first Shapiro step $I_{1}=0.55 \mathrm{~mA}$. It corresponds to an amplitude of the microwave current in the detector of $I_{\omega} \approx 1.8$ $\mathrm{mA}$ and a delivered power of about $5 \mu \mathrm{W}$.

\section{Mixing Two aUtonomous FFOs}

In the first experiments the signals from two freerunning autonomous FFOs were mixed in the small SIS array detector. The frequencies of the FFOs were individually tuned by adjusting the dc bias current and the applied dc magnetic field so that their difference frequency was within the band of the intermediate frequency (IF) amplifier connected to the SIS mixer array. The room temperature IF amplifier (center frequency; $f_{\mathrm{IF}}=1.5$ $\mathrm{GHz}$, bandwidth, $\Delta B_{\mathrm{IF}}=600 \mathrm{MHz}$ ) had an effective noise temperature of $100 \mathrm{~K}$ referred to the SIS junction array.

With this set-up we have detected the mixed signal up to a FFO frequency of $450 \mathrm{GHz}$. The integral (half power, full width) linewidth of the two FFOs was as low as 750 $\mathrm{kHz}$ at $280 \mathrm{GHz}$, see Fig. 5. The results confirm the rather similar experiments done by Zhang et al. [6]. The inherent problem with this kind of measurements is that one may find an unrealistic small integral linewidth because most external disturbances are common-mode to the two FFOs placed on the same chip.

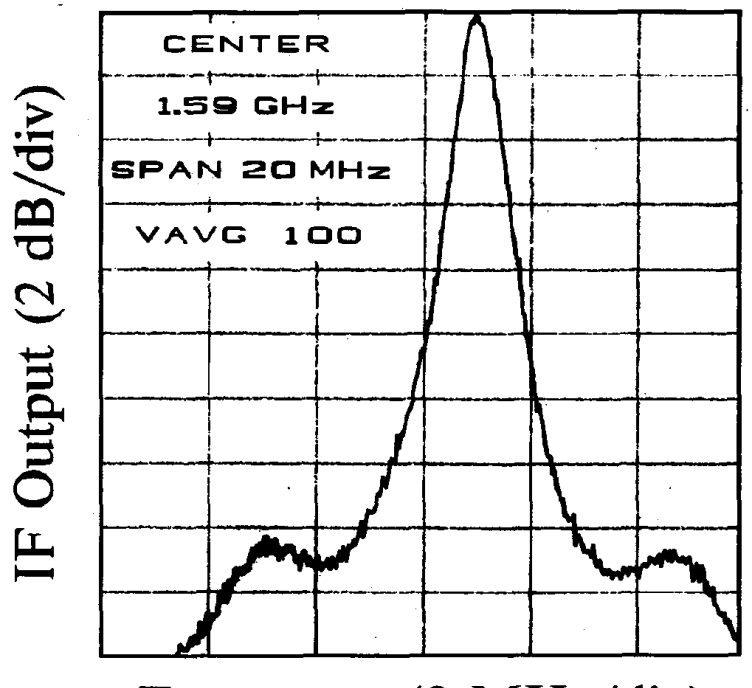

Frequency $(2 \mathrm{MHz} / \mathrm{div})$

Fig. 5. Integral linewidth of two autonomous FFOs at $280 \mathrm{GHz}$ (see text)

\section{FFO AS HARMONIC MULTIPLIER}

In order to measure the linewidth of a single FFO at different frequencies we used a scheme in which a signal from an external frequency-locked room temperature Gunn oscillator (frequency: $f_{\mathrm{G}}=56-74 \mathrm{GHz}$, linewidth: $\Delta f_{\mathrm{G}} \leq$ $80 \mathrm{kHz}$ ) was applied to FFO1 via the fin-line antenna (" 8 " in Fig. 1) and the three-step Chebyshev stepped stripline transformer (" 9 " in Fig. 1) both designed for $70 \mathrm{GHz}$ center frequency. As a result rf-induced Shapiro steps with "usual" voltage spacing, $\Delta V=f \Phi_{0}$, could be generated in the IVC of FFO1.

. The applied frequency, however, is much lower than the maximum plasma frequency of FFO1, $f_{p 0}>200 \mathrm{GHz}$ and the fundamental microwave signal cannot propagate inside the FFO1 tunnel junction. Nevertheless, from the rf-induced steps in the IVC of the SIS array at voltages corresponding to higher harmonics of the applied signal it is inferred that higher harmonic signals (generated by the non-linearity of the FFO1) reach the SIS detector. For small levels of applied power the third harmonic signal (frequency $3 * f_{\mathrm{G}} \approx 220 \mathrm{GHz}$, which roughly coincides with half of the design frequency for the transformer ("12") and the SIS mixer tuning-out inductance) dominantly affects the detector. At higher power levels (where the critical current of the FFO1 and thus the plasma frequency is suppressed) zero-crossing Shapiro steps appear and also a signal at $f_{\mathrm{G}}$ reaches the detector.

The IF frequency ( $f_{\mathrm{IF}}$ resulting from the mixing of the $n$ 'th harmonic of the Gunn oscillator with the FFO2 signal (frequency $f_{\mathrm{FFO} 2}$ ) obeys $f_{\mathrm{FFO} 2}=n * f_{\mathrm{G}} \pm f_{\mathrm{IF}}$ so it is concluded that the FFO1 has successfully been used as a harmonic multiplier [2]. The experimental results 


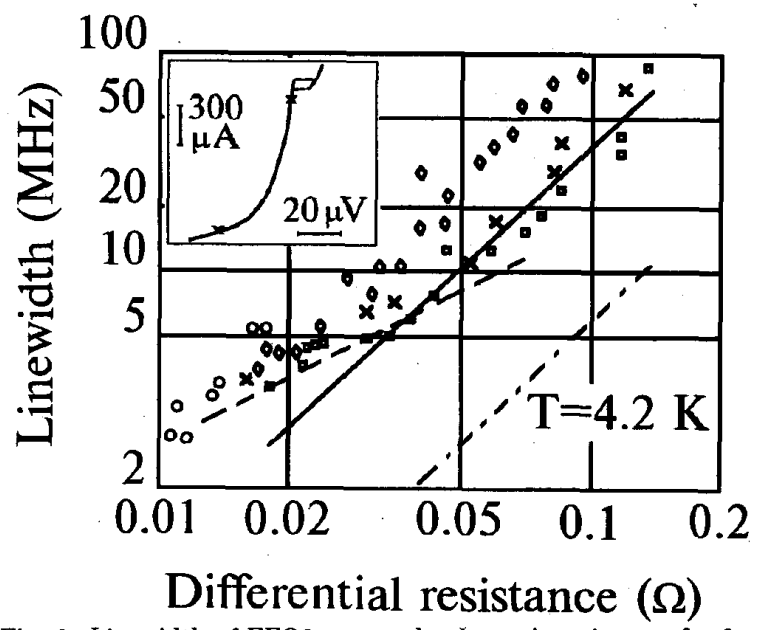

Fig. 6. Linewidth of FFO2 versus the dynamic resistance for four runs recorded with the same sample on different days. Solid line and dot-and-dash line - Eqn. (1) with effective temperature, $T_{\text {eff }}=32 \mathrm{~K}$ and $4.2 \mathrm{~K}$, respectively; dashed line - Eqn. (2) with the amplitude of the low frequency noise current, $I_{f}=0.17 \mu \mathrm{A}$.

showing that the FFO can be phase-locked by injection will be given elsewhere [9]. It was possible to realize harmonic mixing at $n=4,5,6$ and 7 with appropriate Gunn oscillator and FFO2 frequencies.

\section{LINEWIDTh OF A SINGLE FFO}

The observed dependence of the linewidth of the mixed signal, $\Delta f$, on the differential resistance, $R_{d}$, of the FFO2 is shown in Fig. 6. The corresponding IVC of the FFO2 is depicted in the inset. The frequency of the FFO2 was tuned around $350 \mathrm{GHz}$ and we used the 6th harmonic of the frequency-locked Gunn oscillator. The contribution from the Gunn oscillator linewidth $(80 \mathrm{kHz} * 6 \approx 0.5$ $\mathrm{MHz}$ ) can be neglected here and, accordingly, the measured linewidth of the mixed signal can be attributed to FFO2 alone. The $R_{d}$ values used in Fig. 6 were derived from the slope of the dc IVC in the bias point.

One can see from Fig. 6 that the FFO2 linewidth changes proportional to $R_{d}{ }^{2}$ above $5 \mathrm{MHz}$. Below this value $\Delta f$ versus approaches a linear dependence on $R_{d}$.

Presently, no theory exists for the linewidth of the radiation emitted from a FFO. Both theoretical models for the single Josephson junction [10] and the low-damping soliton oscillator [11] give the same dependence of the linewidth on the differential resistance: $\Delta f \propto R_{d}{ }^{2}$ assuming a wideband $\left(f_{\text {noise }} \leq \Delta f\right)$ Nyquist noise spectrum. The linewidth of the high frequency oscillations in a short Josephson tunnel junction in the limit of small junction current fluctuations is given by [10]

$$
\Delta f_{\mathrm{wb}}=2 \pi \frac{R_{d}{ }^{2}}{\Phi_{0}{ }^{2}} e I_{d c} \operatorname{coth}\left(\frac{e V_{d c}}{2 k_{B} T_{\text {eff }}}\right)
$$

where the subindex on $\Delta f_{\mathrm{wb}}$ refers to the wideband noise spectrum with effective temperature $T_{\text {eff. }} V_{d c}$ and $I_{d c}$ is the $\mathrm{dc}$ voltage and current in the bias point.

The best fit (solid line in Fig. 6) to Eqn. (1) using the experimental values for $R_{d}, V_{d c}$ and $I_{d c}$ is obtained for $T_{\text {eff }}=32 \mathrm{~K}$, which is approx. eight times the physical temperature, $T=4.2 \mathrm{~K}$ (dot-and-dash line in Fig. 6 . It should be noted that in some experiments with the same sample (for apparently the same experimental conditions) the $\Delta f \propto R_{d}{ }^{2}$ dependence is still observed but a $T_{\text {eff }}$ as large as $90 \mathrm{~K}$ ( $\diamond$ in Fig. 5) was needed to obtain the best fit.

Low frequency noise with a cut-off frequency much smaller than the width of the spectral line $\left(0 \approx f_{\text {noise }} \ll\right.$ $\Delta f)$ e. g. from external disturbances (bias supplies, temperature fluctuations, hum, $1 / \mathrm{f}$ noise, etc. ) may be directly converted and give rise to excess noise. A low frequency current noise with amplitude, $I_{f}$, will increase the linewidth proportional to $R_{d}$ :

$$
\Delta f_{\mathrm{lf}}=\frac{2}{\Phi_{0}} I_{f} R_{d}
$$

The corresponding dependence for $I_{f}=0.17 \mu \mathrm{A}$ is shown in Fig. 6 by the dashed line fitting the experimental results well for $\Delta f$ below $5 \mathrm{MHz}$.

\section{REFERENCES}

[1] T. Nagatsuma, K. Enpuku, F. Irie, and K. Yoshida, J. Appl. Phys. 543302 (1983); see also Part II-IV in: J. Appl. Phys. 563284 (1984); J. Appl. Phys. 58441 (1985); J. Appl. Phys. 631130 (1988).

[2] A. V. Ustinov, J. Mygind, and V. A. Oboznov, J. Appl. Phys. 721203 (1992).

[3] A. V. Ustinov, T. Doderer, R. P. Huebener, J. Mygind, V. A. Oboznov, and N. F. Pedersen, IEEE Trans. on Appl. Supercond. 32287 (1993).

[4] V. P, Koshelets, A. V. Shchukin, S. V. Shitov, and L. V. Filippenko, IEEE Trans. on Appl. Supercond. 32524 (1993).

[5] V. P. Koshelets, A. V. Shchukin, S. V. Shitov, L. V. Filippenko, G. M. Fisher, and J. Mygind, Physica B 194-196 81 (1994).

[6] Y. M. Zhang, D. Winkler, and T. Claeson, Appl. Phys. Lett. 623195 (1993), see also Proc. Fourth Int. Symp. on Space Terahertz Techn. , Los Angeles, USA pp. 485-499 (1993).

[7] An. B. Ermakov, V. P. Koshelets, S. A. Kovtonjuk, S. V. Shjtov, IEEE Trans. on Magn. 272642 (1991).

[8] V. P. Koshelets, S. A. Kovtonyuk, I. L. Serpuchenko, L. V. Filippenko, and A. V. Shchukin, IEEE Trans. on Magn. 273141 (1991).

[9] V. P. Koshelets, A. V. Shchukin, S. V. Shitov, I. L. Lapytskaya, and J. Mygind, "Spectral linewidth of autonomous and injection locked flux flow oscillators", submitted to Phys. Rev. B. (1994).

[10] K. K. Likharev, Dynamics of Josephson Junctions and Circuits, Gordon and Breach Science Publishers, New York (1986).

[11] E. Joergensen, V. P. Koshelets, R. Monaco, J. Mygind, M. R. Samuelsen, and M. Salerno, Phys. Rev. Lett. 491093 (1982). 